\title{
Aux prises avec les vibrations du social : retour sur le practice turn en relations internationales
}

Grappling with the vibrations of the social: A return to the practice turn in International Relations

Julien Pomarède

\section{(2) OpenEdition Journals}

\section{Édition électronique}

URL : http://journals.openedition.org/conflits/19314

DOI : $10.4000 /$ conflits. 19314

ISSN : $1777-5345$

Éditeur :

CCLS - Centre d'études sur les conflits lilberté et sécurité, L'Harmattan

Édition imprimée

Date de publication : 8 août 2016

Pagination : 151-164

ISBN : 978-2-343-10075-3

ISSN : 1157-996X

\section{Référence électronique}

Julien Pomarède, «Aux prises avec les vibrations du social : retour sur le practice turn en relations internationales », Cultures \& Conflits [En ligne], 102 | été 2016, mis en ligne le 08 août 2016, consulté le 30 mars 2021. URL : http://journals.openedition.org/conflits/19314 ; DOI : https://doi.org/10.4000/ conflits. 19314 


\section{Aux prises avec les vibrations du social : retour sur le practice turn en relations internationales}

\section{Julien POMAREDE}

Julien Pomarède est assistant d'enseignement au département des Sciences politiques de l'Université Libre de Bruxelles et effectue une thèse de doctorat en science politique/relations internationales auprès $d u$ centre Recherche et enseignement en politique internationale (REPI). S'appuyant sur une récente observation participante de plusieurs mois au quartier général de l'OTAN à Bruxelles, ses recherches portent sur une sociologie des pratiques contre-terroristes dans le contexte otanien. Julien a publié plusieurs travaux dans Cultures \& Conflits, Études internationales et le Journal of Intervention and Statebuilding.

Trine Villumsen Berling, The International Political Sociology of Security: Rethinking Theory and Practice, Londres, Routledge, 2015.

Vincent Pouliot, International Security in Practice: the Politics of NATO-Russia Diplomacy, Cambridge, Cambridge University Press, 2010.

Mark Salter, Can Mutlu (dir.), Research Methods in Critical Security Studies: An Introduction, Londres, Routledge, 2013.

Johanna Siméant (dir.), Guide de l'enquête globale en sciences sociales, Paris, CNRS éditions, 2015.

T 'analyse de l'international par le prisme des pratiques a fait surgir un Limportant débat dans la recherche récente en sciences politiques et sociales, à tel point que l'on parle d'un «practice turn » ${ }^{1}$. Les chercheurs y

1. Nous tenons à remercier vivement Emmanuel-Pierre Guittet, Barbara Delcourt, Christophe Wasinski et Cristina Stănculescu, ainsi que les évaluateurs anonymes pour leurs précieux commentaires. 
revisitent par la sociologie politique les objets et domaines d'investigation des relations internationales pour proposer une alternative aux approches théoriques bien connues en la matière. L'intégration européenne, la diplomatie, la sécurité, la stratégie, les politiques environnementales, l'expertise ${ }^{2}$ sont réinterprétées par ce practice turn qui, dans les grandes lignes, entend dépouiller la recherche des catégories préconstruites par lesquelles la discipline des relations internationales s'est historiquement constituée, pour ré-encastrer l'intelligibilité du global dans les espaces sociaux et/ou discursifs par lesquels ce dernier advient et se présente comme réalité empirique. En d'autres termes, il s'agit de défaire l'international d'un statut préconstitué, qui le fait exister en tant que réalité immanente et en dehors de toute intervention de ceux qui l'étudient. Cette démarche repose sur deux principaux enjeux.

Le premier est théorique. L'expression «théorie de la pratique » peut quasiment s'assimiler à un oxymore. Le but de cette approche étant de partir du point de vue des acteurs (l'émique), tenter d'en tirer une théorie peut prendre la forme d'une imposition de catégories heuristiques sur l'expérience des populations étudiées. De là proviendrait le risque d'exercer une déformation sur les chainons qui génèrent les réalités faisant l'objet du regard scientifique. Cette problématique devient plus saillante à l'international, tant cette échelle a historiquement fait l'objet de réifications (notamment par le concept d'anarchie ou d'identité), amenant à la penser avec des lunettes qui lui seraient propres. Sur ce point, les recherches poststructuralistes des années 1990 ont fait le lit du renouveau actuel de ce questionnement. Les poststructuralistes parlent alors de «théorie en tant que pratique ( «theory as practice »). La théorie est avant tout un fait de théorisation. Il s'agit d'un processus actif qui participe à la fois à la sélection des enjeux internationaux devant être légitimement pris en compte par le monde académique ainsi qu’à la définition de la manière de les traiter ${ }^{3}$. Le (néo)réalisme est principalement visé. Par la séparation qu'ils établissent entre théorie et monde réel, les tenants de ce courant participent à la reproduction du projet politique moderne. Ils reconduisent l'idée des Lumières d'un sujet connaissant qui, grâce à l'immanence de sa rationalité, est en capacité de capturer le réel « tel qu'il est vraiment », voire de le contrôler ${ }^{4}$. C'est tout le sens des généalogies respectivement opérées par Richard Ashley ou Jens Bartelson sur la souveraineté. Son émergence coïncide avec celle de la figure de l'homme souverain, capable de modeler le monde à son image 5 .

2. Pour une synthèse des champs d'application des pratiques en RI, voir notamment : Adler Nissen R., Bourdieu in International Relations: Rethinking Key Concepts in IR, Routledge, London, 2013.

3. Zalewksi M., " “All these theories yet the bodies keep pilling up": Theory, Theorists, Theorising ", in Smith S., Booth K., Zalewksi M., International theory: positivism and beyond, Cambridge, Cambridge University Press, 1996, p. 351.

4. George J., Discourses of Global Politics: A Critical (Re)Introduction to International Relations, Boulder, Lynne Rienner, 1994, p. 42.

5. Ashley R., "Living on Border Lines: Man, Poststruturalism, and war », in Der Derian J., Shapiro M., International/Intertextual Relations: Postmodern Readings of World Politics, New York, Lexington Books, 1989, p. 365. 
C'est pourquoi Bartelson parle même d'une « théorie souveraine de la souveraineté 6 ", en référence au fait que le (néo)réalisme produit un savoir politique qui place la rationalité humaine au centre du déroulé de l'histoire.

Le second est d'ordre méthodologique. Voir l'international comme le résultat de pratiques concrètes pose la question des moyens d'accès aux processus et à ceux qui les fabriquent, en particulier pour les recherches attenantes aux milieux confidentiels. Ce questionnement est né d'un débat plus fondamental entre les poststructuralistes et les constructivistes autour du rôle du discours dans la fabrication du monde social. Les premiers accordent une place centrale à l'intertextualité et au discours en admettant qu'ils permettent d'épuiser sur le plan analytique l'ampleur des conditions d'apparition des pratiques. Les constructivistes plus matérialistes considèrent au contraire que le social n'est pas réductible à sa forme discursive. Les énoncés sont à considérer comme des "visibilités ", c'est à dire des "figures portées à la lumière 7 ». Le pouvoir voit et se donne à voir dans des énoncés rendus visibles. Dès lors, il faut « casser les visibilités » des énoncés pour en retrouver toute la logique de production et surtout la mécanique de leurs effets réels. Si l'on n'étudie que la partie visible des énoncés, on n'étudie que ce que le pouvoir donne à voir. Dès lors, les constructivistes (principalement d'inspiration bourdieusienne comme on le verra plus bas) proposent justement de passer en deçà de la partie lumineuse des énoncés en les liant aux dispositions individuelles acquises dans le temps afin de mieux rendre compte des transformations des pratiques. Le discours est alors un acte d'autorité socialement situé : «Les régimes d'énoncés n'épuisent pas les régimes de visibilité. [...] Il y a disjonction entre parler et voir, entre l'énonçable et le visible. Mais il y a une médiatisation et cette médiatisation passe par l'incorporation disciplinaire, par l'babitus qui génère à un moment donné, dans une situation donnée un système de correspondance 8 ». Faire ce pari en relations internationales impose dès lors des méthodes de terrain bien spécifiques afin de passer en-dessous du seuil de visibilité de l'ordre du discours. Comment et par quels moyens peut-on détecter l'international par le biais des pratiques et lorsqu'on fait appel à elles ? Bien plus, dans le cadre du recueil et du traitement des données, dans quelle mesure interviennent la « débrouille » et le «bricolage » dans la lecture par les pra-

6. Bartelson J., A Genealogy of Sovereignty, Cambridge, Cambridge University Press, 1995, p. 17.

7. Deleuze G., Foncault - Les formations historiques, Cours du 12 Novembre 1985 - 2, Université Paris 8. Disponible sur : http://www2.univ-paris8.fr/deleuze/article.php3? id_article $=415$.

8. Bigo D., "Sociologie politique de l'international : une alternative (French)", Cultures E Conflits, En ligne, Articles inédits, 2008, consulté le 2 juin 2016. URL : http://conflits.revues.org/1175. Notons également que l'intérêt actuel pour le practice turn sur le plan méthodologique relève aussi du développement de l'approche ethnographique en RI. Voir notamment sur ce point les travaux de D. Ambrosetti sur le Conseil de sécurité de l'ONU, d'Y. Buchet de Neuilly sur la politique étrangère européenne, d'I. Neumann sur la diplomatie, de C. Cohn et H. Gusterson sur les professionnels de l'armement nucléaire ainsi que ceux de J. Der Derian sur la guerre virtuelle/vertueuse. 
tiques d'un domaine d'étude souvent difficile d'accès et pouvant générer des affects particuliers ?

\section{L’hétérogénéité du Practice turn}

Les quatre ouvrages de notre corpus sont un point d'entrée utile pour effectuer un retour sur ce practice turn : comment la littérature inspirée des pratiques se place-t-elle par rapport à ces enjeux et, de là, quelle valeur ajoutée peut-on en déduire pour la recherche en relations internationales ? Notre idée principale est que l'apport des pratiques est le plus riche et innovant lorsque l'effort est fait de se détacher de l'idée selon laquelle l'international doit faire l'objet de conceptualisations qui lui seraient propres. Dans le cas contraire, les résultats obtenus peuvent l'être sans nécessairement faire appel aux pratiques. Ainsi, notre corpus est un miroir montrant que le practice turn constitue un ensemble hétérogène en termes d'apport et d'originalité, à la fois sur le plan théorique et méthodologique. Deux tendances s'observent parmi nos quatre ouvrages.

La première est celle de l'insertion des pratiques dans les codes classiques de la discipline des RI. Cette démarche s'incarne dans les ouvrages de Vincent Pouliot et Trine Villumsen Berling. Tous deux font appel à la théorie des champs de Bourdieu. Les débats théoriques et méthodologiques novateurs des deux auteurs entamés dans les premiers chapitres de leur livre résistent peu à leur application empirique et à leur proximité avec des approches déjà très employées, notamment celle du constructivisme conventionnel (ou dominant) ${ }^{9}$ en RI.

Pouliot étudie la normalisation sous tension de la diplomatie OTANRussie depuis la fin de l'inimitié bipolaire. Il part de l'état des rapports politiques au Conseil OTAN-Russie (NRC) en 2006. L'institution existe sur la base d'une dynamique duale, où l'exclusion acceptée du recours à la force entre les deux acteurs induit aussi une méfiance mutuelle dans leurs desseins respectifs. Pouliot se fixe l'objectif de faire la sociogenèse de ce constat, en proposant l'argument de rapports diplomatiques normalisés mais ponctuellement très tendus. Il fait l'hypothèse selon laquelle les multiples frictions maillant la diplomatie OTAN-Russie post-Guerre froide proviendraient d'un phénomène d'bysteresis par lequel s'effectuerait la résurgence de «l'habitus de grande puissance russe » («Russian Great power habitus») alors même que la nouvelle structure du «champ de la sécurité internationale » telle qu'édictée par l'OTAN après la bipolarité place la Russie dans une situation objectivement dominée.

9. L'expression est reprise de Macleod A., « Les études de sécurité : du constructivisme dominant au constructivisme critique », Cultures $\mathcal{E}$ Conflits, $n^{\circ} 54,2004$, pp. 13-51. 
De son côté, Berling analyse la reconfiguration des luttes pour la définition légitime de la sécurité européenne après la Guerre froide. La pénétration dans le «champ de la sécurité européenne » de nouveaux acteurs comme les think tanks, tournés vers l'interprétation de la scène internationale par les sciences sociales, aurait provoqué une reconfiguration des rapports de pouvoir s'y déroulant, le capital militaire ayant notamment perdu de sa valeur face à l'émergence du capital scientifique et social. L'OTAN tenterait notamment sa reconversion par l'adoption d'un discours civilisationnel empreint d'idées dotées d'un crédit scientifique (paix kantienne, choc des civilisations) et par le financement de programmes scientifiques (Science for peace and security program).

La deuxième tendance, observable à travers les ouvrages respectivement dirigés par Johanna Siméant et Mark Salter/Can Mutlu, opère au contraire un recentrage sur les pratiques elles-mêmes. L'appareillage théorique et méthodologique ne sert que de point d'appui en second plan à une démarche axée sur une approche inductive. L'ensemble des contributions de l'ouvrage coordonné par Siméant suit la voie d'une banalisation de l'international par le réencastrement de celui-ci dans les propriétés sociales des espaces et acteurs dont les échanges produisent du global. L'enjeu de ce livre est d'appréhender l'originalité de cette démarche dans l'analyse de secteurs aussi divers que la diplomatie, l'Union européenne, l'import/export des savoirs de gouvernement ou encore la circulation internationale des biens culturels et des élites étudiantes. La deuxième partie de l'ouvrage renforce l'argument d'une nonspécificité heuristique de l'international en proposant un recueil de textes d'auteurs contemporains qui se sont penchés sur ce domaine d'étude à travers la diversité des outils fournis par les sciences sociales. Ces extraits sont répartis en quatre parties : "Théories et construction de l'objet ", "Lieux ", «Enquêtes» et « Pistes».

L'ouvrage coordonné par Salter et Mutlu va sensiblement dans le même sens. Dans des chapitres très courts, une trentaine d'auteurs livrent, au travers d'une tonalité personnelle, la complexité et l'apport de leur expérience de recherche propre dans la perspective d'une reconstitution des productions sécuritaires par le bas. Ces dernières sont abordées à travers quatre grandes parties qui recoupent les principaux substrats empiriques et méthodologiques abordés : l'ethnographie, la pratique, la corporalité et la matérialité.

Dans ce qui suit, nous prendrons le temps de développer la dichotomie que l'on vient d'établir afin d'étayer l'argument que nous émettons sur l'hétérogénéité du practice turn. Nous nous réfèrerons successivement aux enjeux théoriques et méthodologiques. 


\section{Enjeux d'une (sur)théorisation de la pratique et de la mise en visibilité du social de l'international}

Dans cette réflexion théorique, on évoquera d'abord le rapport qu'entretiennent les éléments du corpus à la théorisation des pratiques dans l'appréhension de l'international pour ensuite se pencher sur les objectifs poursuivis par la complexification du social inhérente à l'emploi d'une telle démarche.

Sur le premier point, on trouve deux manifestations principales dans le corpus étudié. Les ouvrages de Pouliot et de Berling forment une première position, qui consiste en une lecture théorisée des pratiques. Revenons sur l'emploi par Pouliot du concept d' "habitus de grande puissance russe ». L'auteur indique dans son chapitre théorique que "l'babitus est historique 10 ", soulignant par-là que les effets de ces dispositions intégrées ne peuvent être compris qu'à travers un détour par l'épaisseur de leur historicité. Il apparaît dans l'application empirique de l'babitus que l'auteur donne l'ascendant à une interprétation théorisée du concept. La proposition d'un habitus national se fait par le biais à la fois d'une historicisation relativement brève de telles dispositions ${ }^{11}$ et de l'application du concept à une entité aussi vaste que complexe : un État-nation. Par-là, Pouliot entend rassembler les multiples dispositions agentielles irriguant la politique étrangère russe dans l'idée d'un savoir inarticulé incarnant la "grande puissance russe». En cela, cet emploi de la notion d'habitus se révèle assez proche de l'utilisation constructiviste conventionnelle de la notion plus classique de "culture stratégique 12 », qui considère les options diplomatico-militaires comme provenant d'une identité nationale inscrite dans les routines d'État.

On retrouve aussi une vision théorique de la pratique dans l'ouvrage de Berling. Le concept de «capital » sert de point d'ancrage à l'analyse pour lire les transformations $\mathrm{du}$ "champ de la sécurité européenne » après la Guerre froide et la configuration des luttes qui s'y renouvellent pour l'autorité sur la définition légitime de la sécurité européenne. L’auteure utilise le capital pour mettre en lumière les opportunités et les contraintes propres à un moment de perturbation d'un espace social, ainsi que la provenance et l'effet relationnel de la mobilisation de ressources. Ce qui est surtout le cas des stratégies de reconversion de l'OTAN, l'organisation étant poussée à adapter la morphologie de ses capitaux face à la pénétration significative d'autres acteurs.

Les implications empiriques de l'autonomie prêtée par l'auteure au capital scientifique dans les transformations du champ de la sécurité européenne sont d'importance et peuvent donc faire ici l'objet d'une discussion approfondie.

10. Pouliot V., op. cit., p. 31.

11. Ibid., p. 177.

12. Glenn J. «Realism versus Strategic Culture: Competition and Collaboration? », International Studies Quarterly, vol. 11, n 3, 2009, p. 535. 
La fin de la Guerre froide voit sans doute moins une nouvelle forme d'interaction science/pratique se jouer, que son insertion potentielle dans un enjeu plus fondamental qu'est la restructuration des imaginaires politiques (ou des cartographies) de la scène internationale, à savoir les (re)configurations de l'inimitié ${ }^{13}$. Il apparaît que l'identification de l'impact structural des capitaux en jeu relève plus d'un choix de l'auteure que d'une évaluation en profondeur des modalités de production des ressources de pouvoir qui maillent les luttes pour la définition légitime de la sécurité internationale. Certes, les indices relevés par l'auteure par son analyse notamment de la NATO review existent et révèlent dans une certaine mesure les stratégies de réaffirmation de l'OTAN. Néanmoins, une lecture plus ancrée conduirait probablement à introduire dans l'équation otanienne deux dimensions essentielles de la légitimation de l'Alliance dans sa vie post-bipolaire : les pratiques interventionnistes et techno-stratégiques, à travers lesquelles l'OTAN capitalise sur son rôle dans une nouvelle cartographie essentiellement militarisée des insécurités.

Les deux autres ouvrages du corpus fournissent une vision différente de la pratique sur le plan de leur rapport à la théorie. La priorité analytique est donnée aux interactions humaines elles-mêmes, la théorie ne servant qu'à mieux rendre compte d'une complexité sociale visualisée par une démarche inductive.

Dans le livre dirigé par Siméant, on comprend que l'international n'a pas de raison d'être appréhendé par des outils théoriques qui lui seraient propres. La question est posée dès l'introduction : "Pourquoi les sociologues devraient-ils s'arrêter de faire des sciences sociales dès lors que leurs objets changent d'échelle ? 14 ». Les contributions tendent à établir que la théorie ne sert qu'à consolider et à approfondir l'observation de structures de relations spécifiques. Sur le «capital international », A.C. Wagner et B. Réau établissent que «le débat sur l'existence ou non d'un capital spécifique par rapport aux autres capitaux doit ainsi être déplacé. Il faut en effet se garder d'essentialiser le capital international. Les ressources internationales efficientes ne peuvent être définies en elles-mêmes : elles sont spécifiques à chaque champ ${ }^{15}$ ».

La partie consacrée à l'approche ethnographique dans le Salter \& Mutlu offre une perspective similaire. On y voit que la théorisation ne fait que suivre les jalons d'analyses issues de confrontations directes avec le terrain. H.L. Johnson explique par exemple ce qu'elle a tiré de son immersion dans des camps de réfugiés pour la compréhension des effets de pouvoir générés par la

13. Bigo D., "Grands débats dans un petit monde », Cultures E Conflits, n 19-20, 1995, pp. 748.

14. Siméant J., «Introduction - Face à l'international. Textes et pratiques de l'enquête en sciences sociales ", in Siméant J. (dir.), op. cit., p. 6.

15. Wagner A.C., Réau B., « Le capital international : un outil d'analyse de la reconfiguration des rapports de domination ", in Siméant J. (dir.), op. cit., p. 46. 
globalisation du contrôle des frontières sur la construction interindividuelle des identités migratoires ${ }^{16}$.

Le second pas de notre discussion théorique nous emmène vers la question suivante : pourquoi et dans quels buts complexifier l'international par la mise en avant des relations sociales qui le produisent ?

Une similarité s'observe à nouveau entre l'ouvrage de Pouliot et de Berling. On dira que la complexification que ces auteurs font des objets internationaux s'avère relative, dans la mesure où ils priorisent l'insertion de ces derniers dans les catégories heuristiques qu'ils mobilisent. À cet égard, Pouliot place la réalité ontologique de la pratique diplomatique au niveau agentiel des entités étatiques et à celui du système international pour ce qui est du structurel. Les premiers chapitres théorique et méthodologique contiennent une réflexion intéressante sur l'apport de la sociologie bourdieusienne en RI (par rapport au rationalisme et aux démarches représentationnelles notamment). L'analyse empirique de Pouliot reste toutefois proche d'une conception de l'international relevant du constructivisme conventionnel.

La haute échelle ontologique à laquelle Pouliot place son débat rappelle la théorie structuraliste d'Alexander Wendt, dont le cour est l'explication de la fabrication d'intérêts nationaux dans le cadre d'interactions interétatiques durables, elles-mêmes conditionnées par un environnement normatif plus large ${ }^{17}$. Proche de cet axe, Pouliot établit en effet des liens de causalité directs entre les élargissements de l'OTAN et la résurgence d'une politique étrangère russe moins collaborative, voire plus agressive vis-à-vis des puissances occidentales. Ces interactions étant enveloppées par la structure (post-bipolaire) renouvelée du «champ de la sécurité internationale » plaçant la Russie dans une situation de domination qu'elle n'accepte justement plus à partir des premiers élargissements de l'Alliance en 1994. De là, on peut en fait en tirer la complexification des « cultures anarchiques » de Wendt ${ }^{18}$. Par la mise en visibilité des variations diplomatiques OTAN-Russie tolérée par l'application à haute échelle de la sociologie de Bourdieu, Pouliot montre l'existence d'un entre-deux anarchique, ni kantien, ni lockéen, dans lequel l'exclusion normalisée du recours à la force côtoie d'importantes tensions diplomatiques (différend du Kosovo en 1999 et du conflit russo-géorgien en 2008). Ainsi, la démonstration de Pouliot se place à un degré de complexification de la pratique diplomatique et en tire des résultats qui peuvent se révéler proches des conclusions formulées plus classiquement par le constructivisme conventionnel.

16. Johnson H.L., « Listening to migrant stories », in Salter M., Mutlu C. (dir.), op. cit., pp. 6771.

17. Wendt A., Social Theory of International Politics, Cambridge, Cambridge University Press, 1999.

18. Ibid., pp. 246-312. 
La question de la complexification se pose également chez Berling, mais sous un autre angle. L'hypothèse de l'auteure consistant à établir un renversement de la valeur des capitaux dans le " champ de la sécurité européenne » après la Guerre froide a pour enjeu de rendre plus complexe la compréhension de la restructuration des formes d'autorité dans l'espace étudié. Comme nous l'avons brièvement évoqué, la démarche amène en fait l'auteure à flouter les frontières heuristiques entre complexification et surestimation du changement. À nouveau, il nous paraît pertinent de débattre les conclusions de Berling. Montrer que des acteurs prennent de l'importance grâce à la légitimation de nouvelles formes de ressources est une réalité, mais affirmer l'autonomie de ces ressources et la conséquence de leur impact sur la trajectoire d'autres acteurs paraît un autre ordre de réalité ${ }^{19}$. Ainsi, la démonstration de Berling va sans doute loin lorsqu'elle établit que l'entrée dans le champ de la sécurité européenne des think tanks provoque un recentrage de l'OTAN sur un capital scientifique. Paradoxalement, la complexification de l'analyse des significations de la sécurité européenne sur la base de l'étude des capitaux tend à simplifier la densité des particularités historiques des acteurs impliqués dans la mobilisation de ces mêmes ressources. Ainsi, un accent plus net mis sur les dispositions politiques et stratégiques qui fondent l'OTAN conduirait par exemple sans doute Berling à conclure à une reconversion plus conservatrice que ce qu'elle suggère, et à une relativisation de la capacité de transformation doxique de certains «nouveaux» acteurs.

L'ouvrage coordonné par Siméant témoigne de l'existence d'une tout autre vision et d'un tout autre apport des modes de complexification de l'international par le biais de l'emploi des pratiques. Le rendu complexe du global est à la fois vu comme une pré-condition heuristique, mais aussi comme le cœur du travail de recherche lui-même. Dans les contributions, le recours à la prosopographie est récurrent pour comprendre comment la trajectoire des acteurs contribue à l'internationalisation de leurs objets d'activité. D. Georgakakis et A. Vauchez considèrent ainsi le concept de champ comme l'opportunité d'ouvrir les différentes boîtes noires de l'Union européenne. La vertu complexificatrice de l'emploi du champ est dès lors de déconstruire l'UE non plus comme une entité, mais comme un processus actif, celui de l'européanisation. Dès lors, s'ouvre au chercheur la diversité des logiques professionnelles qui tapissent l'évolution quotidienne de l'espace européen 20.

19. C'est un des apports du concept de « conjoncture fluide» de M. Dobry. Voir : Dobry M., Sociologie des crises politiques, Paris, Presses de Sciences Po, 2009.

20. Vauchez A., Georgakakis D., «Le concept de champ à l'épreuve de l'Europe », in Siméant J. (dir.), op. cit., pp. 210-211. 


\section{Traquer, situer et « bricoler » l'international}

Dans cette partie d'ordre méthodologique, nous aborderons d'abord les différentes manières par lesquelles les éléments du corpus entendent « traquer » et « situer » l'international pour ensuite nous intéresser aux diverses « improvisations » méthodologiques nécessaires ou non au recueil et au traitement des données, en particulier dans le cadre de recherches en milieux confidentiels.

Sur le premier point, une perspective claire est posée par l'ouvrage de Pouliot. L'international est d'emblée posé. Élever l'argument au niveau d'habitus nationaux et d'un "champ de la sécurité internationale » amène à une lecture du global à partir de lui-même. La pratique sert à complexifier cette catégorie reprise comme telle dans l'analyse. Chez Pouliot, c'est donc la pratique qui va à la rencontre de l'international, ceci permettant à l'auteur de fournir l'argument selon lequel il existe des pratiques internationales en soi.

Une position plus nuancée est offerte par l'ouvrage de Berling, notamment par la délimitation empirique du « champ de la sécurité européenne ». Dans cette entreprise, Berling problématise les dichotomies classiques des RI qui peuvent, selon elle, trouver des concrétisations plus fondées empiriquement en étant traitées avec plus de souplesse. L'auteure réfléchit ainsi sur les « niveaux d'analyse », les « frontières géographiques de l'Europe », et la différenciation " high and low politics». Ces dimensions sont vues comme les axes à cadrer pour reconstruire l'espace effectif du « champ de la sécurité européenne ». À nouveau, Berling lit ces éléments à travers le concept de capital. Les modalités de leur activation et leur capacité de frontiérisation sont visibles par le constat des limites dans lesquelles se ressentent les effets des ressources en jeu pour la définition légitime de la sécurité européenne ${ }^{21}$. La manœuvre est convaincante. Berling met effectivement en avant l'existence d'un illusio commun réunissant des acteurs aux intérêts divers, celui de la définition légitime de la sécurité européenne. Dans ce choix de méthode, on constate aussi une tendance chez l'auteur à poser l'argument d'un international a priori. Les remarques faites précédemment sur l'ascendance donnée au capital par rapport aux dispositions dans l'analyse de Berling nous conduisent à aussi formuler un commentaire quant aux conséquences de ce choix sur notre sujet de discussion. Intéressons-nous à la manière dont l'auteur envisage l'agence de l'UE. La concurrence entre cette dernière et l'OTAN est principalement travaillée dans le cadre de la quête d'autonomie (capital) militaire de l'UE. Dès lors, voir l'UE sous un tel angle conduit Berling à internationaliser a priori son débat, en envisageant des rapports entre un acteur homogène comme l'UE et l'OTAN. La capacité de l'UE à s'affirmer comme acteur clef de la définition de la sécurité européenne s'est structurée de manière sans doute plus cruciale à

21. Berling T.V., op. cit., p. 31. 
travers des dispositifs à la fois fort différenciés professionnellement et nonnécessairement militaires ${ }^{22}$. L'UE constitue un assemblage complexe et relativement transnationalisé en termes de ressources professionnelles. Délier cette entité de la notion de capital pour effectuer un retour sur les dispositions de la multiplicité des agents impliqués dans la conduite sécuritaire de l'UE aurait sans doute amené un travail de questionnement sur les frontières du « champ de la sécurité européenne » (et donc des espaces de déploiement de l’international) plus dense.

L’ouvrage coordonné par Siméant fournit probablement le questionnement le plus poussé et le plus dénaturalisant de la détection de l'international. Chacune des contributions a à cœur de démontrer que ce dernier ne prend forme que dans divers secteurs et objets, ceux qui sont précisément le fait d'une internationalisation. L'international est moins pensé comme un objet en lui-même, que comme un phénomène empirique composé de multiples objets qui, par de complexes processus de transactions sociales prenant place dans des espaces aux enjeux spécifiés, prennent une forme internationalisée. De là, les auteurs concluent en général à une autonomie relative des espaces et capitaux internationaux, car perpétuellement enchevêtrés avec des propriétés sociales élaborées au niveau national. Le recueil de textes qui compose le second bloc du volume rend d'autant mieux compte de la valeur heuristique accordée par les contributeurs à une dispersion de l'international. Siméant affirme ainsi en référence au texte de J.F. Bayart sur le comparatisme international : «Il est vital de réencastrer l'analyse de l'international dans le fonds général des sciences sociales, par exemple en développant une conception de la comparaison qui n'arase pas l'épaisseur historique des objets étudiés 23 ». Loin de constituer une entrave à la compréhension de l'international, la réinsertion de ce dernier dans l'immense appareillage des sciences sociales est le creuset de son approfondissement. Ici, on dira que c'est plutôt le global qui vient aux sciences sociales.

L'ouvrage de Salter et Mutlu partage sensiblement la même perspective sur les manières d'entreprendre l'identification de l'international. Si l'interrogation sur le sujet n'est pas aussi saillante que dans l'ouvrage dirigé par Siméant, l'utilisation «fidèle » des auteurs classiques en sciences sociales amène les contributions vers une localisation de l'international par le prisme des pratiques elles-mêmes. C'est notamment le cas de H. Hugues, qui propose une lecture de la mise en sécurité du changement climatique en tant que «pratique d'écriture » ("practice of writing »). En prenant le cas de l'International Panel on Climate Change (IPCC), l'auteure établit que celui-ci constitue un champ de pratique transnational pourvu d'identités professionnelles assez

22. Jeandesboz J., «Labelling the "neighbourhood": towards a genesis of the European neighbourhood policy ", Journal of International Relations and Development, vol. 10, $\mathrm{n}^{\circ}$ 4, 2007, pp. 387-416.

23. Siméant J., «Théories et construction de l’objet », in Siméant J. (dir.), op. cit., p. 221. 
solides pour y faire naître un intérêt de lutte pour la domination sur un discours sécuritaire. Plusieurs sous-unités administratives entrent en compétition dans l'IPCC pour détenir "l'autorité scientifique dans et sur la procédure d'évaluation 24 »de l'institution.

Notre second questionnement méthodologique relatif au «bricolage » dans l'appréhension du terrain fournit deux types d'éclairages dans notre corpus. Le premier est incarné par l'ouvrage de Berling et de Pouliot, dans lesquels on constate à la fois que l'enquête de terrain est d'une utilité relative pour leur démonstration et que le «bricolage » n'est pas nécessairement de mise pour appréhender l'international par les pratiques.

Berling nous donne à voir une application « ordonnée » de la théorie de la pratique bourdieusienne. L'auteure n'ayant pas recours à des techniques d'enquêtes particulières (observation participante ou entretiens), mais plutôt à des méthodes d'analyse de contenus, l'ouvrage retient l'option de l'analyse des pratiques sans devoir faire appel à des arrangements particuliers dans le recueil des données.

Pouliot quant à lui propose une réflexion sur la manière dont l'entretien peut servir de substitut à l'impossibilité de l'observation participante au Conseil OTAN-Russie (NRC). L'auteur utilise les entretiens pour photographier un état de fait politique prévalant en 2006 au NRC. De ces entretiens émerge un premier chapitre empirique intéressant, «The Logic of Practicality at the NATO-Russia Council », qui rend compte de la nature intériorisée d'une diplomatie méfiante entre Russes et Occidentaux. C'est à partir de ce constat qu'il déconstruit dans la suite de l'ouvrage les séquences passées ayant permis l'émergence de ces relations politiques. En fait, la valeur ajoutée des entretiens ne se reflète véritablement que dans l'exposé théorique et dans ce premier chapitre consacré au NRC. Dès lors que l'auteur remonte historiquement pour expliquer cet état de fait, l'analyse relève du constructivisme conventionnel par lequel la vertu que l'auteur prête aux entretiens pour lire la pratique n'est pas nécessaire aux besoins de la démonstration.

À partir de leurs recherches respectives, J. Siméant, V. Lickert et F. Pouponneau livrent également quelques éléments et ficelles pour approcher les milieux confidentiels 25 . Une palette de solutions est fournie pour mener une enquête viable sans bénéficier du schéma idéal de l'observation participante. Est abordée notamment la construction d'entretiens productifs. L’objectif prêté à ces derniers étant de tracer les contours des identités professionnelles parties à des interactions autour d'enjeux diplomatiques ou humanitaires, il est par exemple conseillé de se constituer une expertise sur les sujets

24. Hughes H., « The practice of writing », in Salter M., Mutlu C. (dir.), op. cit., p. 95.

25. Siméant J., Pouponneau F., Lickert V., "Échelles, récifs, bureaux - terrains du politique à l'international », in Siméant J. (dir.), op. cit., pp. 26-32. 
abordés en interviews afin de mieux cerner les différences entre groupes sociaux impliqués. L'innovation est également conseillée dans le traitement des sources. F. Pouponneau explique ainsi qu'il a cherché du côté des «sujets et corrections de travaux de l'ENA ", afin de voir si des examens portant sur la rédaction de télégrammes diplomatiques ne contiendraient pas des informations de première main sur le dossier du nucléaire iranien.

Sur le plan du «bricolage », un des apports les plus essentiels du Salter \& Mutlu est de s'interroger sur les opportunités et les contraintes relatives aux situations de recherche parfois si particulières des études critiques de sécurité. Tout en admettant la place nécessaire au resserrage théorique d'une recherche, l'ensemble des auteurs plaident pour la reconnaissance heuristique des divers improvisations et sentiments qui fondent le quotidien d'une recherche de type critique. Par opposition à la tonalité désincarnée de la plupart des manuels de méthode en RI, les contributions prennent des dimensions très personnelles, voire tournent parfois à la confidence. C'est d'ailleurs une des raisons potentielles qui pourrait expliquer pourquoi l'ouvrage est titré « research methods" et non pas «methodologies». La contribution de J.P. Crane-Seeber en est un bon exemple. Son chapitre porte sur la production des masculinités de guerre parmi les soldats américains déployés en Irak. Relatant son expérience dans les bases militaires américaines ou dans les villes au sein desquelles elles étaient situées, l'auteur évoque l'apport heuristique des diverses tensions normatives et des transformations personnelles générées par la proximité quotidienne avec les soldats. Le chercheur nous parle ainsi du « learning by feeling 26 ».

Le plaidoyer que constitue cet ouvrage pour les arrangements méthodologiques constitue également sa limite la plus importante. En critiquant la rigidité scientifique des grands courants en RI, l'ouvrage tombe dans l'excès inverse : celui de placer quasiment au cœur de la recherche le bricolage méthodologique. Si les situations atypiques auxquelles peuvent donner naissance les études critiques (en particulier les démarches ethnographiques) laissent de la place pour l'improvisation (certes nécessaire et créative), il reste que la rigueur méthodologique reste un des garde-fous qui établissent la frontière entre le regard scientifique et les acteurs que celui-ci analyse, entre la sociologie de métier et la sociologie spontanée. À trop bricoler sans formaliser et s'interroger sur les inconscients de sa recherche, on en reviendrait à reproduire la logique des négociations et arrangements quotidiens que les acteurs du monde social opèrent. L'argument de fond de l'ouvrage doit donc être appréhendé de manière relative, en particulier pour les plus jeunes étudiants qui se lancent dans les sciences sociales de l'international. Comme le disaient Bourdieu, Chamboredon et Passeron, «c'est peut-être la malédiction des sciences de l'homme que d'avoir affaire à un objet qui parle 27 ». Un constat qui est d'au-

26. Crane-Seeber J.P., « Learning by feeling », in Salter M., Mutlu C. (dir.), op. cit., pp. 72-75.

27. Bourdieu P., Chamboredon J.C., Passeron J.C., Le métier de sociologue, Paris, Mouton de Gruyter, 2005 [1968], p. 56. 
tant plus prégnant lorsque les coins les moins éclairés de l'international ouvrent leurs portes, donnant ainsi naissance à un sentiment utile mais trompeur : «J'y suis donc je comprends ».

\section{Les pratiques de l'international : des « mains dans le cambouis » aux « fastes » de l'élévation théorique}

Pour conclure, notre question de départ portait sur l'utilité des pratiques pour lire l'international. Notre lecture croisée offre deux conclusions principales. Premièrement, le corpus confirme l'hétérogénéité que peut recouvrir le practice turn. Tant dans le degré d'innovation que dans le statut des analyses par rapport aux grands courants en RI, le pivot heuristique que l'on attribue habituellement à l'emploi des pratiques s'avère relatif. Sur ce plan, l'offre scientifique est ainsi large, à la fois pour les chercheurs qui penchent plutôt pour l'inclusion de la pratique dans la discipline internationaliste, ou pour ceux qui souhaitent une lecture plus dénudée de la fabrication du global. Ensuite, que l'on opte pour la première ou l'autre tendance, on retiendra que l'originalité de la lecture de l'international par les pratiques réside en partie dans le fait d' « oublier » ou du moins de parfois « laisser de côté » la théorie et la méthode dans la quotidienneté de la recherche. Dans une certaine mesure, réinsérer la qualité interprétative du chercheur en écartant modérément et de manière séquentielle un moulage théorique serré, permet justement de reconstruire les chaînons pratiques des faits sociaux et, de là, de déduire une architecture théorique forte, originale, car mise au service d'une démonstration empirique concrète.

C'est sans doute là qu'est l'apport le plus décisif des sciences sociales pour l'international. Il ne réside pas tant dans le fait de vouloir à tout prix démontrer qu'elles « ouvrent de nouvelles perspectives », mais plutôt dans celui d'accepter la modestie de leur quintessence : autant que possible, partir de là où les objets partent, aller là où les objets vont. C'est paradoxalement à partir du cambouis des vibrations du social que les fastes théoriques se révèlent dans toutes leurs nuances.

En définitive, le practice turn semble s'associer à un acte performatif de cloisonnement plutôt qu'à un acte de libération scientifique. Il semble reconstruire les balises théoriques et méthodologiques positivistes qui flèchent avec force le chemin de la découverte, alors que les sciences sociales et plus spécifiquement les pratiques donnent la chance au chercheur de se perdre dans des environnements qu'il peut reconstituer sans nécessairement trop en châtier la réalité de leur trajectoire. Si les vibrations du social restent un défi d'importance à relever dans la compréhension de l'international, force est de constater que l'international lui-même reste un défi aux tentatives de captation de l'agir humain. 